\title{
Using Clustering Techniques to Optimize Panel Grouping for Large PV Arrays with Non-uniform Orientation and Shading Obstructions.
}

\author{
Andy McNeil ${ }^{1}$ \\ ${ }^{1}$ Independent Researcher, Oakland, CA, USA
}

\begin{abstract}
This paper demonstrates a clustering method for grouping PVs of arbitrary orientation affected by nonuniform local shading. For a project with 44,000 PV panels cladding doubly curved roof surfaces, every panel has a unique orientation. In addition, clerestory windows cause non-uniform near-field shading. The combination of curvature and shading causes every panel to receive a different amount of irradiance at any time. The PV panel with lowest output (lowest irradiance) in a MPPT string limits the output of the whole string. The method for grouping 44,000 PV panels into 296 MPPT strings affects the total annual energy producing. The PV array grouped with clustering generated $7.7 \%$ more energy annually compared to the same array grouped in a simple grid.
\end{abstract}

\section{Introduction}

Figure 1 shows a roof plan of the building to have a PV clad roof. There are 52 diamond or triangle shaped roof bays. Each roof bay is curved in two dimensions. Clerestory windows along the intersection between some roof bays cause shadowing on some panels of the lower bay.

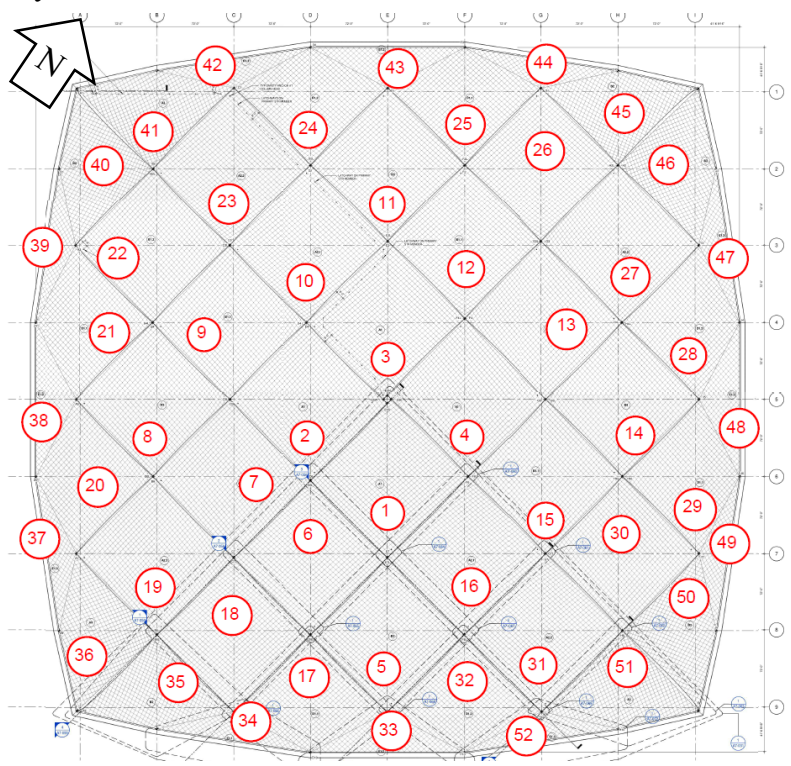

Figure 1: Roof plan showing bay numbering scheme.

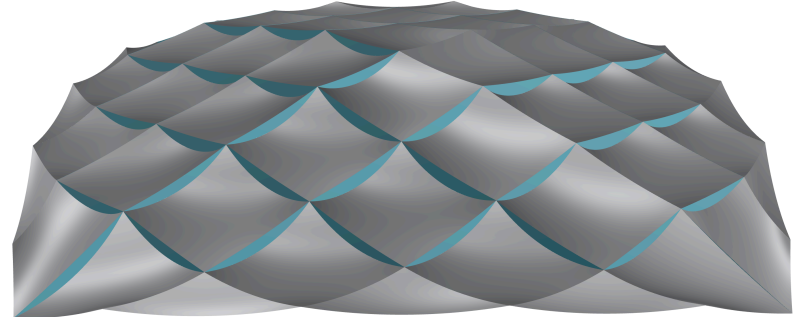

Figure 2: Rendering of CAD model of roof surfaces. Gray surfaces are tiled PV panels. Cyan surfaces are clerestory windows. Clerestory windows are vertical, and not visible in plan view.

When estimating the annual energy produced by the rooftop PV we are faced with the following challenges:

- clustering 1,200 PV panels per roof bay into eight groups

- simulating 1,200 unique PV orientations per roof bay

- accounting for shading from clerestories

These challenges were overcome for this project using a combination of k-means clustering, PVWatts for PV simulation, and Radiance for time varying shade factors.

Clustering is a means of dividing objects into groups based on shared similarity. There are many techniques for clustering, the most common being k-means (Jain et al., 1999). The k-means algorithm clusters objects into groups based on distance from the object to the cluster centroid in n-dimensional Euclidean space. Objects are placed into the cluster with the closest centroid (in a Voronoi like manner), and cluster centroids are modulated to minimize the summed squared distance between objects and the cluster centroid. Other clustering techniques consider measures such as distance to nearest object in a cluster (rather than cluster centroid), or consider aspects other than distance to define clusters, such as Gaussian lobe fitting.

PVWatts, is perhaps the most full featured means for simulating annual PV energy production (Dobos, 2014). The online PV simulator accounts for many conditions affecting PV output, including hourly PV operating temperature and angle of incidence correction for PV module cover reflectance. However PVWatts can only account for shading with a single shade factor that is uniformly applied to the whole year.

Radiance is a lighting simulation program that can perform ray-tracing simulations on arbitrary geometry generated in CAD software (Ward and Shakespeare, 
1998). Radiance's daylight coefficient simulation capability and matrix based coefficient storage and arithmetic operators allows for efficient annual simulation of irradiance.

\section{Background}

Substantial research exists on performance of partially shaded PV arrays and electrical methods for optimizing output. Patel and Agarwal (2008) and Sera and Baghzouz (2008) developed models for simulation of partially shaded PV arrays. Dolara et al. (2013) performed an expiramental investigation on partial shading providing data useful for further developing simulation models for PV output.

Wang and Hsu (2011) simulated partially shaded PV systems, modelling different electrical connection architectures under various types and levels of partial shading. They identified total-cross-tied (TCT) configuration to preform best by most measures. However their analysis focuses on electrical connections in a small system of PVs with no need for electrical subgrouping.

The assumption for most research is that all PV panels in a system have uniform orientation, receiving the same insolation when not shaded. BiPV on curved building surfaces causes variation in insolation across a PV system. Cheng's master's thesis (2009) developed methods for optimizing the shape of curved BiPV surfaces to increase output.

Existing research largely focuses on developing models for partially shaded PV systems and evaluating electrical configurations to optimize output under partially shaded conditions, and optimizing curved shapes to maximize PV production. I was unable to find any research dealing specifically with the task of grouping PV panels in a very large PV system to reduce occurance and severity of partial shading insolation varition within groups.

\section{Methods}

- Step 1 - Group PVs based on irradiance profile

- Step 2 - Estimate hourly energy production of PV roof

The least producing panel on the string limits the output of each MPPT. The optimal zoning groups panels with similar production profiles.

\section{PV Grouping}

Each roof bay has 2-8 MPPTs, depending on the size of the bay. Each panel on the roof is connected in a string to one of the MPPTs. Each MPPT can serve $35-312$ panels. However, each MPPT has a $15 \mathrm{~kW}$ max output. When the power produced by panels exceeds the max output for the MPPT, the additional power is lost. To avoid exceeding $15 \mathrm{~kW}$ the maximum number of panels is reduced to 220. Based on these constraints, we restricted group sizes between 35 and $220 \mathrm{PV}$ panels per MPPT.

The PV panels in a bay were grouped based on irradiance profiles for the following six sunny days extracted from TMY weather data: Dec, 23, Jan 21, Feb
25, Mar 24, Apr 21, May 21, Jun 27. Using a text editor, the hourly data for the six selected days, and the header were extracted from the site TMY data file, and placed into a truncated weather file. The Radiance program 'gendaymtx' created a sky matrix from the truncated weather file. The $-\mathrm{O} 1$ option is used with gendaymtx to generate a skymatrix of total solar radiance. Following is the Radiance command for generating the sky matrix for panel grouping.

\section{gendaymtx -m 4 -01 sky/sunnydays.wea > sky/sunnydays.smx}

To start, a script places an analysis point is placed at the center of each PV panel in the Rhino model. The coordinates of the points are exported to a text file. Then to find the facing angles of each panel, the Radiance program 'rtrace' was used to trace a ray originating 10 $\mathrm{mm}$ above the analysis point in the $-\mathrm{Z}$ direction. Rtrace returned the normal direction of the intersected surface (the PV panel). The analysis point and surface normal are written to a separate text file for analysis.

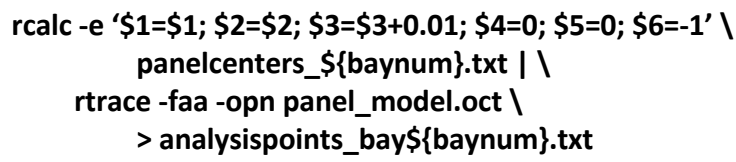

Then the following 'rfluxmtx' command creates a daylight coefficient matrix using the analysis points. The daylight coefficient matrix uses the Reinhart M4 sky subdivision, with 2305 sky patches.

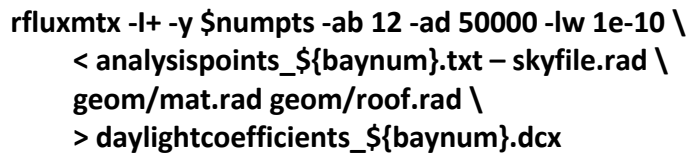

The daylight coefficient matrix and the sky matrix are multiplied using the Radiance program 'rmtxop'. The output of rmtxop is an irradiance matrix containing 73 irradiance values (each hour over the six sunny days) for every panel. The 73 irradiance values are considered to be a profile that characterizes patterns of the irradiance observed by each PV panel on sunny days.

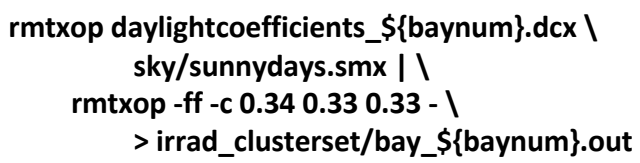

The PV panels are grouped into natural clusters based on the irradiance profile using k-means clustering with octave (an open source computing environment similar to Matlab). The k-means clustering algorithm groups elements based on proximity in n-dimensional Euclidean space. Of the clustering methods considered, k-means was selected because clustering PV panels based on sum of squares difference in irradiance seemed to be consistent with our goal of grouping panels with similar temporal irradiance profiles. The following octave script was used to perform the initial k-means clustering on the 73-dimensional irradiance vectors. 
\#! /usr/local/bin/octave -qf
pkg load statistics

infile $=\operatorname{argv}()\{1\}$;

numclust $=\operatorname{str} 2$ num $(\operatorname{argv}()\{2\})$;

outfile $=\operatorname{argv}()\{3\}$;

A=dlmread(infile);

[numpts,numdims] =size(A);

[idx, centers]=kmeans( $A$, numclust $)$;

csvwrite(outfile,idx);

With k-means clustering the user can specify the number of groups to be formed, but cannot constrain the size of each group (neither maximum nor minimum size). Of the other clustering algorithms considered, none allow users to constrain the size of resulting groups. In many of the 52 bays, the clustering result violated the size constraints outlined above. To resolve the cluster size violations, an iterative process reassigned panels to a new group based on Euclidean distance in 73dimensional hyperspace. The iterative process followed the following steps:

1. Compute the centroid of each cluster (in 73dimensional hyperspace).

2. For each group with more than 220 panels, starting with the largest group, move panels furthest from the group centroid to their next closest cluster until there are 220 panels in the group. If no groups exceed the maximum constraint, starting with the smallest group, find panels in the other group closest to the centroid of the small group in other groups and move panels into the small group until the number of panels in the small group reaches 35 .

3. Recompute cluster centroids for the new panel assignments.

4. Move all panels to the cluster with the closest centroid.

5. Check the size of all clusters, if they violate size constraints, then repeat beginning at step 1 .

Although step 4 undoes a lot of the reassignment performed in step 2, it ensures that each panel prefers its assigned cluster to all other clusters in the roof bay. With enough iteration, the cluster centroids shift, and the panel groupings conform to the size constraints. Surprisingly, the general pattern of the initial k-means clusters remains apparent after iterative reassignment.

\section{Annual PV simulation}

The second step is to determine the approximate annual output of the rooftop PV array. We used a combination of PVwatts and Radiance to simulate the annual production of the PV arrays.

PVWatts takes the orientation (azimuth and tilt angles), panel type, size, and location as input and provides annual and hourly energy production as output. PVWatts computes hourly solar irradiance on the PV panel, calculates the reflective losses based on module cover and solar incidence angle, calculates the cell temperature based on environmental and cell parameters, and from the calculates the DC output of the system. The ability to calculate cell temperature based on ambient temperature, wind speed, insolation, and panel characteristics is a major benefit to the PVwatts method.

PVwatts does not have the ability to input shadowing geometry or a shadow schedule, only an annually uniform shade loss, which can be included in the system losses factor (with soiling, age, light-induced degradation, and other loss factors). The roof studied has substantial self-shading for some panels near the clerestory that is non-uniform in time (heavily shaded at some times and not shaded at others). Our method used Radiance simulations to account for temporally nonuniform shadows from the roof geometry.

The hybrid PVWatts-Radiance method starts by calling the PVwatts API to return hourly irradiance and hourly DC output values individually for each panel in a group. The hourly irradiance values were only used to verify consistency between PVWatts and Radiance for unshaded panels. The hourly DC values were put into a Radiance matrix format, where a row represents each PV panel and contains hourly output values for the whole year. The matrix file contains the appropriate header describing the number of rows, columns, and components in the matrix. The following python code uses the requests library to call the PVWatts API and generate a Radiance formatted matrix file for all the panels in a group.

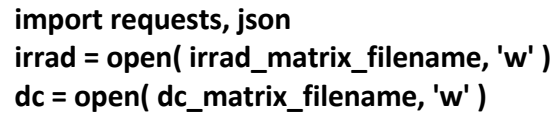

Then we generate a sky matrix for the entire year using the same TMY weather data used by PVWatts and simulate the hourly irradiance for all panels with and 
without obstruction using the Radiance rfluxmtx method described in the previous section. The following commands were used to generate the shaded hourly irradiance values. The unshaded irradiance matrix is generated with the same commands above omitting the underlined model files.

gendaymtx -m 4 -01 sky/Moffett.wea > sky/Moffett.smx

\author{
rfluxmtx -faf -w -I+ -n 8 -y \$numpts -ab 1 -ad 200000 -Iw \\ $1 \mathrm{e}-10 \backslash$ \\ $<$ analysispoints_\$\{baynum\}-\$groupnum\}.txt । \\ - \$skyfile geom/mat.rad geom/roof.rad \ \\ $>$ DCX/shaded_\$\{baynum\}-\$groupnum\}.dcx \\ rmtxop -ff DCX/shaded_\$baynum\}-\$\{groupnum\}.dcx \ \\ sky/Moffett.smx | | \\ rmtxop -ff -c $0.340 .330 .33-1$ \\ $>$ irrad/shaded_\$ $\{$ baynum\}-\$groupnum\}.out
}

Then we multiply the DC output matrix by the shaded obstructed irradiance and divide by the unobstructed irradiance to generate a shadow adjusted hourly DC output for each panel. This matrix operation can be performed with the Radiance program rmtxop as follows:

\section{rmtxop -ff DC/PVwatts_\$ $\{$ baynum\}-\$groupnum\}.out * irrad/shaded_\$\{baynum\}-\$groupnum\}.out 1 / irrad/unshaded_\$\{baynum\}-\$groupnum\}.out | $>$ DC/Adjusted_\$\{baynum\}-\$groupnum\}.out}

The final step is to take the DC hourly output of all panels in a group and estimate the total group output. First we need to reduce the power output of each panel to that of the lowest producing panel in the group. Then we sum the power production for all the panels. The group is reduced to the maximum output of the MPPT $(15 \mathrm{~kW})$ if the group power exceeds that limit. Finally, the group output is multiplied by the inverter efficiency.

\section{Results}

We'll use roof bay number five (Figure 1), as an illustrative example of panel grouping results. Roof bay 5 is predominantly south-east facing, with a clerestory window causing shade on the northwest edge of the bay. Figure 3 shows a rendering illustrating the position of roof by 5 in the roof. Figure 4 shows the panel layout for roof bay 5 , with seven panel positions labeled A-G.

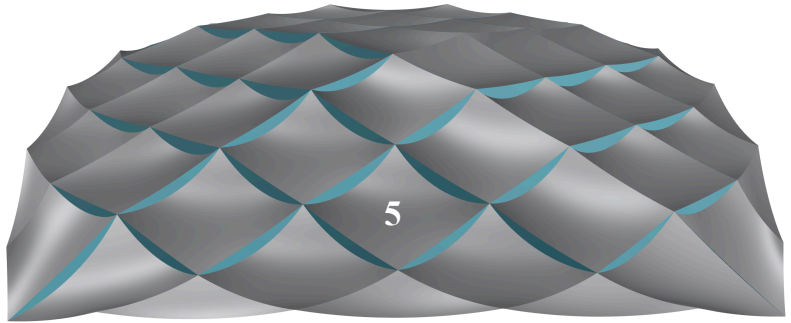

Figure 3: Rendering of CAD model of roof surfaces with roof bay 5 identified.

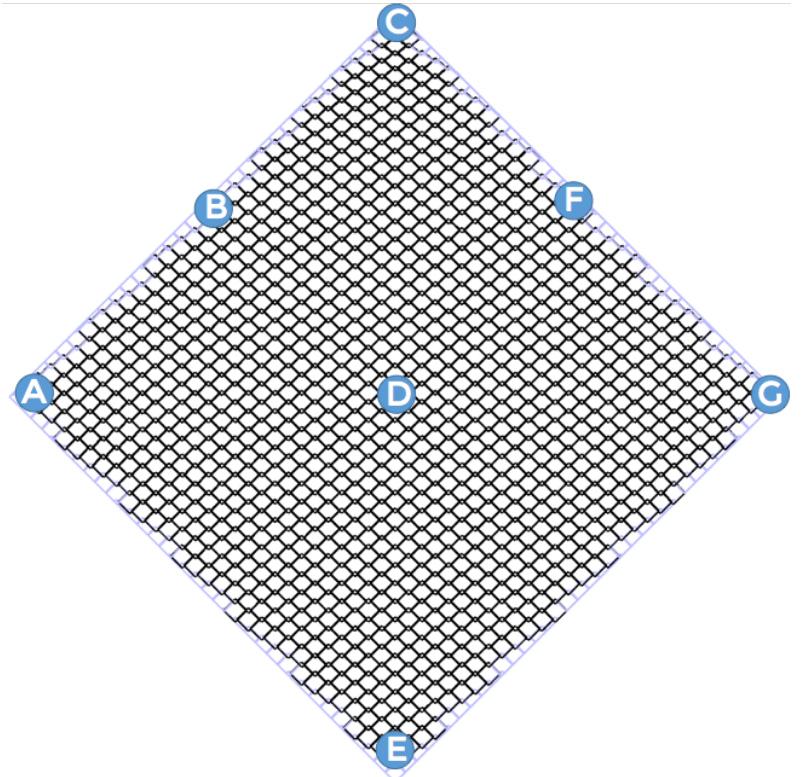

Figure 4: Plan of roof bay 5 with seven test panels labelled A through $G$.

Table 1: Tilt and azimuth angles for the test panels.

\begin{tabular}{|c|c|c|}
\hline Panel & Tilt & Azimuth \\
\hline A & 21 & 39 \\
\hline B & 5 & 138 \\
\hline C & 35 & 147 \\
\hline D & 1 & 336 \\
\hline E & 1 & 159 \\
\hline F & 5 & 154 \\
\hline G & 22 & 261 \\
\hline
\end{tabular}

Table 1 contains orientation and tilt data for the seven selected panels. Tilt is measured from zenith ranging from $0^{\circ}$ for horizontal panel orientation, up to $90^{\circ}$ for vertical panel orientation. Azimuth is arranged so that $0^{\circ}$ is north and $90^{\circ}$ is east.

\section{Panel Grouping}

Figure 5 contains the June 27 portion of the calculated irradiance vector used for clustering for the seven highlighted panels. This figure illustrates the variation that occurs between irradiance on the panels owing the varied orientation and shading from the clerestory window. Panel B experiences the most shading, with a sharp drop in irradiance in the early afternoon. Panel C experiences shading an hour later. Panels A and G have almost the same tilt angle and don't experience shading, but the panel orientation affects the irradiance. Panel A facing north-northeast has peak irradiance in the morning. Panel $\mathrm{G}$ has a west-southwest orientation causing irradiance to peak later in the afternoon. 


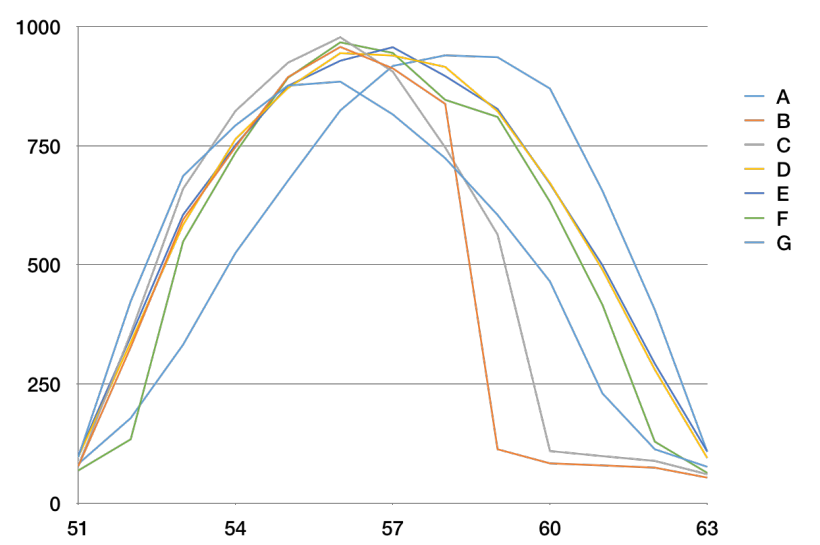

Figure 5: Panel irradiance values (W/m2) for hours 5163 hour in the clustering vector (representing a sunny June 27th).

Figure 6 shows the result of the k-means clustering algorithm for roof bay 5. Each dot represents a PV panel with the dot's color indicating the group assignment. Panels with the same color are grouped together. The orange group exceeds the number of panels that can be on a single MPPT with 541 panels, (an MPPT can only support 220 panels). With $17 \mathrm{PV}$ panels, the purple group is less than the minimum number (35) of panels for an MPPT.
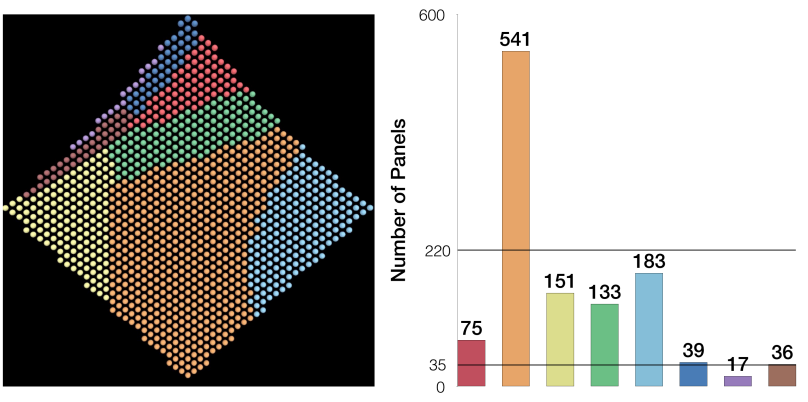

Figure 6: Panel grouping after clustering for bay 5. The group is indicated by colour, and the bar chart on the right shows how many panels are in each group.

All bays required at least one iteration of panel reassignment to meet group size limitations, and 19 of the 52 bays required only one reassignment. On average bays required 17 iterations of reassignment, with a median of 12 iterations. The maximum number of reassignment iterations required by a bay was 62 . Bay 5 required 17 iterations of reassignment to satisfy group size restrictions. Figure 7 shows the panel groupings after iterative reassignment, this bay. The purple group grows, taking some of the blue and brown group. The blue group grows to include the original red group. The brown group expands to encompass much of the original yellow group. The yellow group takes some from the orange group, and the remainder of the orange group splits into two, forming a new red group.

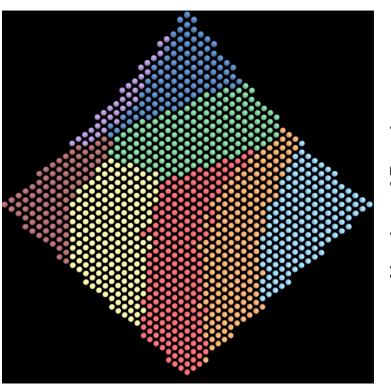

600

Figure 7: Panel grouping after iterative re-assignment of panels to groups.

Figure 8 shows the grouping results for all roof bays after k-means clustering and iterative reassignment steps.

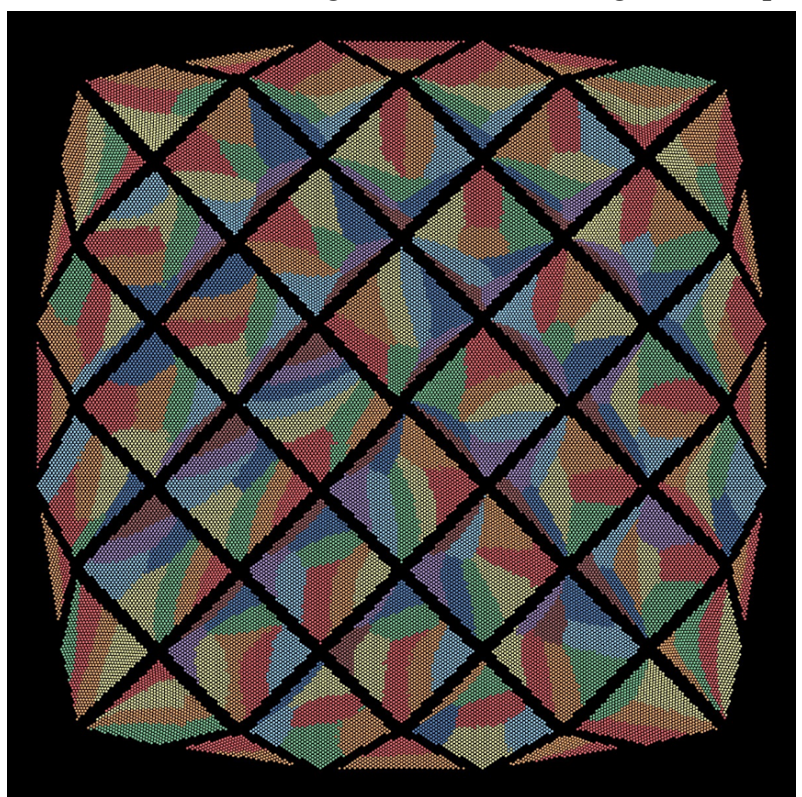

Figure 8: Panel grouping diagram for all bays of the roof.

\section{Annual PV simulation}

First, to verify agreement between PVwatts and Radiance, we generated unshadowed panel irradiance values for the eight panels test panels in bay 5 (Figure 4 A through $G$ ) with both Radiance and PVwatts. Figure 9 contains scatter plots irradiance simulated with the two programs plotted on each axis. Panels with near zero tilt angle have nearly perfect agreement. As the tilt increases, the agreement gets slightly worse, but is within range of acceptability. The disagreement between the PVWatts and Radiance likely stems from the difference in sky model used by each program. Radiance uses the Perez All-Weather sky luminance model (Perez et al., 1993), while PVwatts uses Perez's method for modelling irradiance components from direct and global irradiance (Perez et al., 1990). 

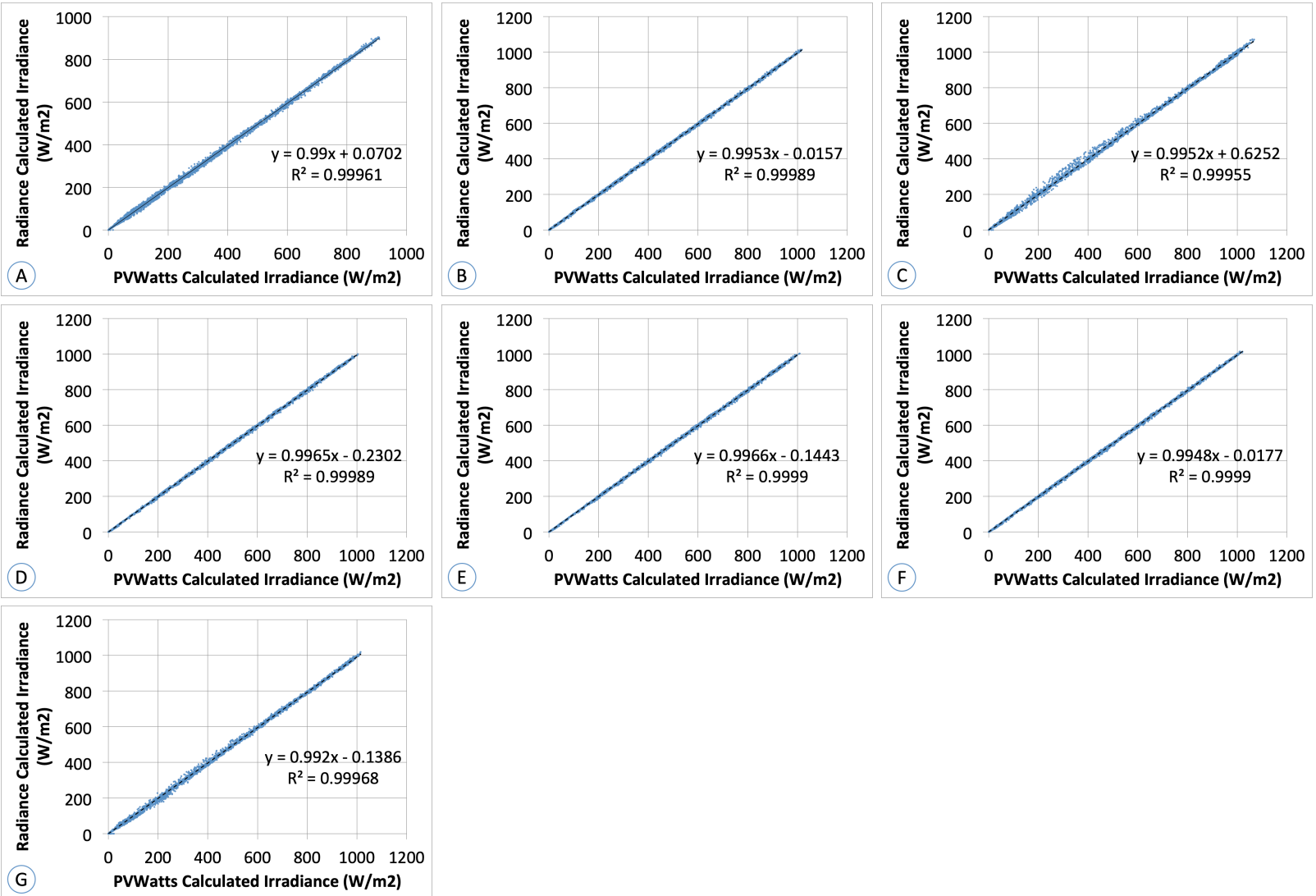

Figure 9: Scatter plots of panel irradiance calculated by PVWatts (x-axis) vs, Radiance (y-axis) for panels A-G
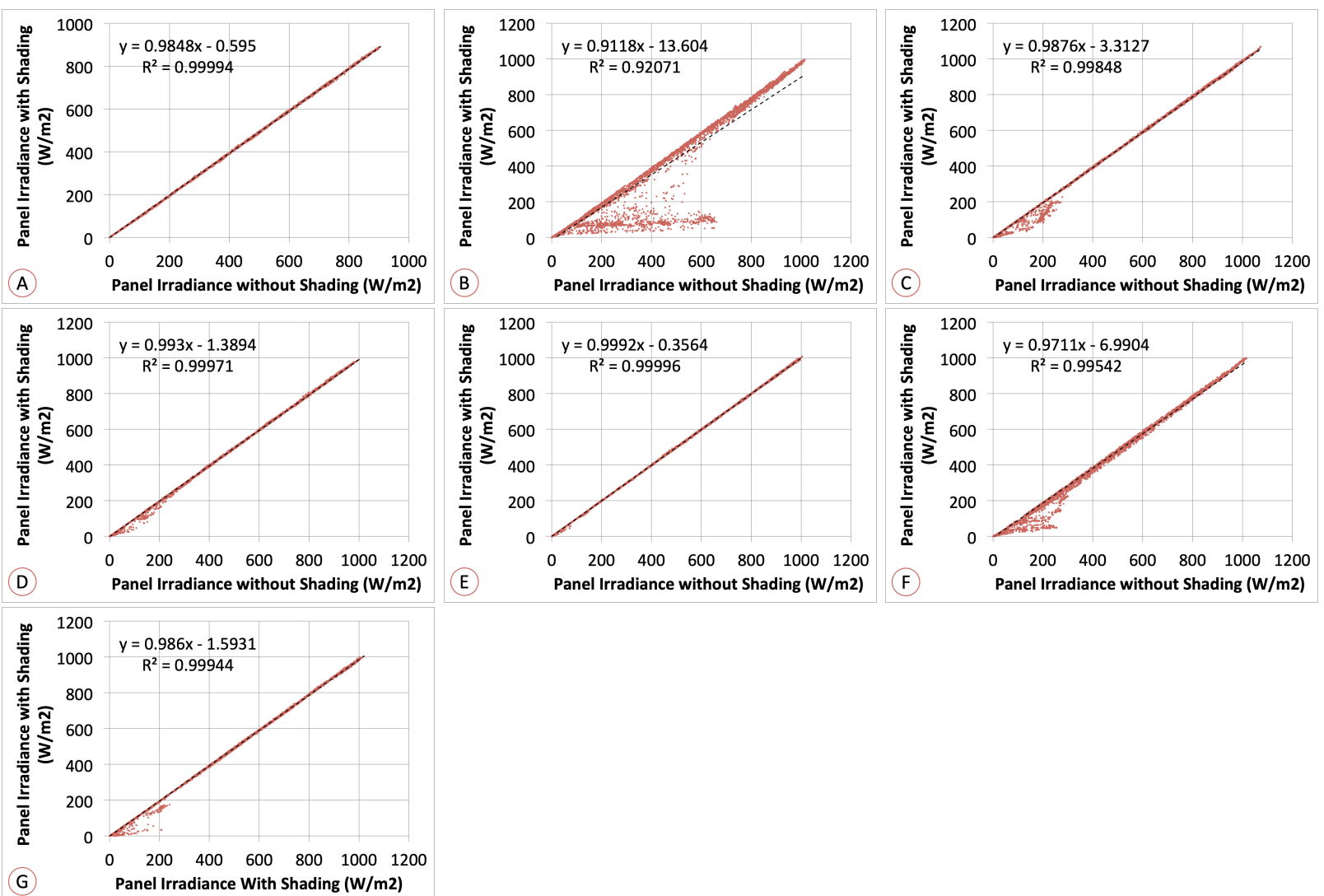

Figure 10: Scatterplots of panel irradiance with and without shading (both calculated using Radiance). 
Then, hourly shade factors are generated by simulating irradiance on the panel in Radiance with and without shading. The ratio of shaded to unshaded irradiance for a panel during an hour is used to modify the dc power output of the panel for that hour. Figure 10 contains scatterplots showing unshaded vs. shaded PV panel irradiance simulated with Radiance for the eight bay 5 test panels. Shading occurs at times where the points fall below the $\mathrm{y}=\mathrm{x}$ diagonal. Panel $\mathrm{B}$ experiences the most shading from the roof, while panel $\mathrm{E}$ and $\mathrm{A}$ experience almost no shading.

We then estimate the annual energy produced by the rooftop PV by applying the described methodology for every panel in every group in every roof bay. Unfortunately confidentiality prevents us from sharing the estimated annual energy production in this paper.

We compared the results using the clustered panels to results using the simple grid-based grouping scheme depicted in Figure 11. The clustering method resulted in a $7.7 \%$ increase in the estimated annual energy produced by the rooftop PV.

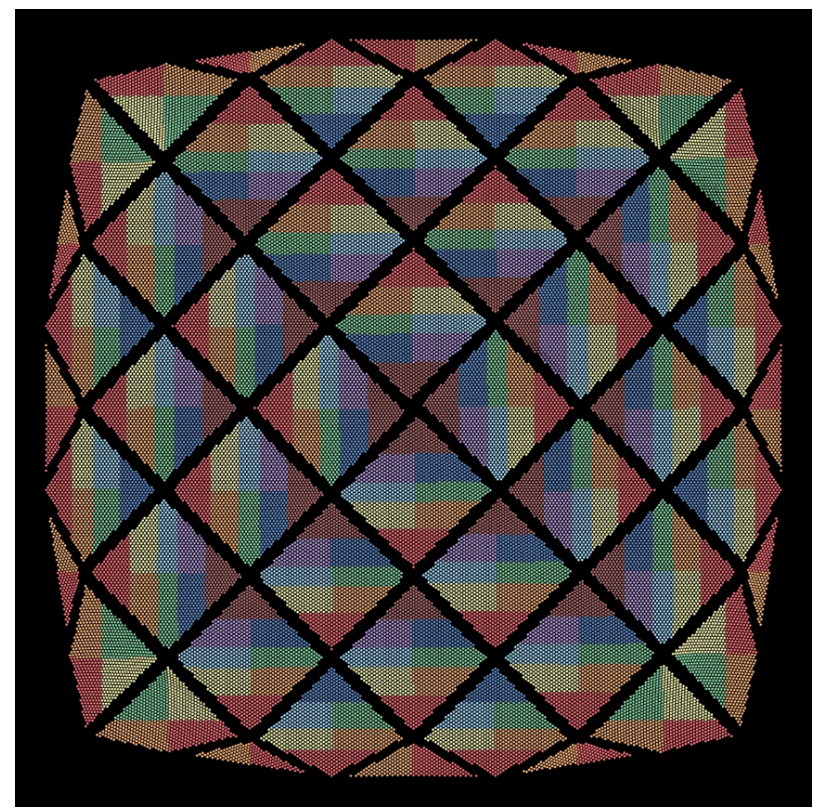

Figure 11: An illustration of the simple grid grouping scheme used as a baseline to determine impact of clustering on annual energy production.

\section{Discussion}

This clustering method is useful for BIPV on curved surfaces and/or PV arrays with non-uniform shading. For a PV array with uniform panel orientation and little to no shading, grouping arrangement has little to no effect on output.

One option that wasn't considered in this study was using dummy panels (or non-connected) panels in heavily shaded regions of the roof, for example panel B in roof bay 5 (see Figure 4 and Figure 10). Including dummy panels reduces the number of panels generating energy, but might increase energy produced by eliminating frequently shaded and therefore lowest performing panels.
Another regret of the author was using sun position at the middle (half-hour) of hourly timesteps for clustering the panels. The author believes this could lead to bias in the grouping divisions along hourly shade lines, which might perform well in hourly energy simulation, but perform worse in reality. A better approach would be to use sub-hourly intervals for clustering, while randomizing sun position within the timestep.

\section{Conclusion}

This paper proposes a method for grouping 44,000 PV panels into groups of 35-220 panels in a way that improves annual energy production. Due to the roof curvature, the PV panels have varying tilt and orientation angles. Clerestories create non-uniform and time varying shading characteristics. These two characteristics make optimal grouping for energy production a challenge.

The demonstrated method formed groups using k-means clustering on hourly irradiance profile vectors generated with Radiance. When the clustering result violated the group size constraint of 35-220 panels per group, an iterative re-assignment process was used to move panels to their next closest group.

The annual energy production was calculated first by generating annual hourly shade coefficients for each of the 44,000 PV panels. This shade coefficient was the ratio of shaded to unshaded irradiance on the panel, simulated with Radiance. The annual hourly shade coefficients were multiplied by hourly DC power from PVWatts simulations. The hourly energy produced per group was determined based on the number of panels in the group and the panel with lowest power for the hour. The total annual energy produced is the sum of energy produced for all the hours for all the groups in all the roof bays.

The annual simulations were performed for two grouping schemes, using the intelligently clustered panels (Figure 8), and with a simple grid based grouping scheme (Figure 11). The grouping scheme developed using k-means clustering produced $7.7 \%$ more energy over the year.

\section{Acknowledgements}

I thank my colleagues at Arup: Varand Balasanian, Joshua Wetzig, Ioni Papaioannou, Tina Sack, Geoff Gunn, and Erin McConahey. I also wish to thank our client, who remains confidential.

\section{References}

Cheng, S. (2009). Curved photovoltaic surface optimization for BIPV: an evolutionary approach based on solar radiation simulation. Doctoral dissertation, UCL (University College London).

Dobos, A.P. (2014). PVWatts version 5 manual (No. NREL/TP-6A20-62641). National Renewable Energy Lab. (NREL), Golden, CO (United States).

Dolara, A., Lazaroiu, G.C., Leva, S. and Manzolini, G. (2013). Experimental investigation of partial shading 
scenarios on PV (photovoltaic) modules. Energy, 55, pp.466-475.

Jain, A.K., Murty, M.N. and Flynn, P.J. (1999). Data clustering: a review. ACM computing surveys (CSUR) 31(3), 264-323.

Patel, H. and Agarwal, V. (2008). MATLAB-based modeling to study the effects of partial shading on PV array characteristics. IEEE transactions on energy conversion 23(1), 302-310.

Perez, R., Seals, R. and Michalsky, J. (1993). Allweather model for sky luminance distributionpreliminary configuration and validation. Solar energy 50(3), 235-245.

Perez, R., Ineichen, P., Seals, R., Michalsky, J. and Stewart, R. (1990). Modeling daylight availability and irradiance components from direct and global irradiance. Solar energy 44(5), 271-289.

Sera, D. and Baghzouz, Y. (2008), October. On the impact of partial shading on PV output power. In WSEAS/IASME International Conference on Renewable Energy Sources, 229-234.

Wang, Y.J. and Hsu, P.C. (2011). An investigation on partial shading of $\mathrm{PV}$ modules with different connection configurations of PV cells. Energy 36(5), 3069-3078.

Ward, G. and Shakespeare, R. (1998). Rendering with Radiance: the art and science of lighting visualization. 